\title{
Traditional Chinese Bitter Flavor Theory: Is There Any Relation with Taste Type II Receptors?
}

\author{
Yu-xin Zhang ${ }^{\text {a\#\#}, \text { Xing Wang }}{ }^{\text {b\#}, \text { Shi-feng Wang }}{ }^{a}$, Yan-Ling Zhang ${ }^{a^{*}}$, Yan-Jiang Qiao ${ }^{a^{*}}$
}

\begin{abstract}
Affiliation
${ }^{\text {a }}$ Key Laboratory of TCM-Information Engineer of State Administration of TCM, School of Chinese Materia Medica, Beijing University of Chinese Medicine, 6 Wangjing Zhonghuan Rd, Beijing 100102, China

b Beijing Key Lab of TCM Collateral Disease Theory Research, School of Traditional Chinese Medicine, Capital Medical University, No.10 Xitoutiao, Outside Youanmen, Beijing 100069, China.
\end{abstract}

\section{* Corresponding author:}

Dr. Yan-Jiang Qiao, Key Laboratory of TCM-Information Engineer of State Administration of TCM, School of Chinese Materia Medica, Beijing University of Chinese Medicine, Beijing 100029, China. Tel.: +86 10 84738620; fax: +86 2184738620.

E-mail address: yjqiao@263.net (Y.-J. Qiao)

Dr. Yan-Ling Zhang, Key Laboratory of TCM-Information Engineer of State Administration of TCM, School of Chinese Materia Medica, Beijing University of Chinese Medicine, Beijing 100029, China. Tel.: +86 10 84738620; fax: +86 2184738620.

E-mail address: collean_zhang@163.com (Y.-L. Zhang)

\# These authors contributed equally to this work. 


\section{Abstract}

Introduction: As a guide principle, traditional Chinese medicine (TCM) theories study the formation mechanism and the application regularity of Chinese herbs, while it is limited by the understanding of the natural properties of the herbs at the cognitive level. As one of important part in TCM theories, Five Flavor theory describes the function of drugs with five tasting adjectives: bitter, sweet, pungent, sour and salty. Methods: In this study, we present reasonable hypotheses that Bitter Flavor theory having instructive significance on account of the relation with bitter taste receptors (taste receptors, type 2, also known as T2Rs). For the lacked crystallized structure of T2Rs, in silico modeling method allows us to visualize the relationship between compounds from TCM showing Bitter Flavor property and T2Rs. In this study, pharmacophore models of T2R1, T2R10, T2R14 and T2R46 have been generated to screen agonists from TCM showing Bitter Flavor property. Results: According to the simulation results, $71.84 \%$ agonists of $\mathrm{T} 2 \mathrm{Rs}$ were derived from medicinal herbs showing Bitter Flavor properties and the experimental evidence of the hits through virtual screening was validated via literature mining. Conclusion: The methods used in this research on bitter taste receptors supports the assumptions underpinning the traditional Chinese Bitter Flavor theory and provides a way to explore the potential science behind the Five Flavors theory of TCM which could be extended to explore other similar problems in TCM theory.

\section{Key Words}

Five Flavors; Taste type 2 receptors; Pharmacophore Model; Data Mining; Pharmacological Efficacy 


\section{Introduction}

Traditional Chinese Medicine (TCM) property theory, evolved from long-term medical practice with the contemporary thoughts and theories in social and nature sciences ${ }^{1}$, is an important pharmacy theory which is based on the physiological, pathological and clinical effect when drugs act on human body. As a guide principle, TCM property theory has played an important role in syndrome differentiation and clinical prescription for thousands of years $^{2,3}$. TCM property theory studies the formation mechanism and the application regularity of TCM properties, including four properties ${ }^{1}$, five flavors ${ }^{4,5}$, meridian entry ${ }^{6}$, toxicity and so on. These various characteristics description of efficiency as drugs effect on human body forms the TCM property theory of the system. Specifically, Five Flavors describe the function of drugs, Four Properties describe the principle of drugs, Meridian Entry describes the position of action and so on. These elements are combined to form a cohesive whole and impact on each other.

However, TCM theory is limited by the understanding of the nature at the cognitive level $^{1}$ and more objective, quantitative references should be added to evaluate the guiding significance. In this direction, we have made big emotional investment to evaluating the Five Flavors theory, one element of TCM theory systems. In Five Flavors theory, five basic tastes have been contained: bitterness, sweetness, sourness, saltiness, and pungency. Previous studies focused on Pungent Flavor ${ }^{4}$, while this research focuses on the Bitter Flavor theory.

According to Five Flavor theory, 'flavor' describes more than the taste feeling of drugs and each of the five flavors has its own particular effect on the body. Bitter Flavor, for example, can reverse the upward motion of $Q i$ in lung, activate $Q i$ and blood motion, dry damp evils and so on. The drugs showing Bitter Flavor property have potentially therapeutic effects in coughs, stagnant blood syndrome, vomiting and so on. Modern research has also 
shown that: taste is not just about taste ${ }^{7}$. The bitter taste receptors have been found a series of effects such as reversing obstruction on airway smooth muscle ${ }^{8}$, therapeutical effect in asthma $^{9,10}$, nutrient sensing in the stomach ${ }^{11}$ and so on, which is consistent with Bitter Flavor theory. Furthermore, there are some agonists of bitter taste receptor showing therapeutical effects. As is reported in Nature Medicine, agonists of these receptors are better than current $\beta 2$-adrenergic bronchodilators in a mouse asthma model, which raise the possibility that bitter compounds, alone or in combination with current therapies, may have a future as bronchodilators $^{12}$. Besides, it is also reported that agonists of T2R3, T2R4, T2R10 and T2R14 induced strong endothelium-independent relaxations, the responses of which are between $82-96 \%$ of maximal relaxations ${ }^{13}$. Even more enticing, 15 clinical drugs were found to activate the $\mathrm{T} 2 \mathrm{R} 14^{14}$ and hypothesis that extraoral bitter taste receptors as mediators lead to off-target effects of any drug with a bitter taste ${ }^{15}$. Therefore, we hypothesize on the basis of research that traditional Chinese Bitter Flavor theory has instructive significance on account of the relationship between compounds from TCM showing Bitter Flavor properties and bitter taste receptors.

\section{Methods}

In this study, natural agonists of T2Rs and the TCM sources with related properties were selected in Table $1^{15}$ according to the following criteria: (a) showing typical structural feature; (b) whose natural plant sources showing Bitter Flavor property.

According to the Bitter Database built in 2012 (http://bitterdb.agri.huji.ac.il/dbbitter.php), taste type II receptors and specific ligands distribution have been summarized in Figure 1, which remind us of some hints about the law of T2Rs. In this study, pharmacophore model of T2R1, T2R10, T2R14 and T2R46, which have been reported possessing more ligands, have been generated to study the relation 
between TCM showing Bitter Flavor property and bitter taste receptors. A pharmacophore can be considered as the highest common denominator of a group of molecules exhibiting a similar pharmacological profile and which are recognized by the same site of the target $\operatorname{protein}^{16}$. A pharmacophore model explains how structurally diverse ligands can bind to a common receptor site. Furthermore, for the characteristic of convenience, fastness and direct vision, pharmacophore models can be used to identify through virtual screening novel ligands that will bind to the same receptor ${ }^{17}$.

Despite recent progress in structure determination of GPCRs, structural data on GPCRs are scarce and crystallized receptor proteins exhibit only low amino acid sequence similarity with T2Rs. Therefore, ligand-based characterization for T2Rs is necessary to understand the relation with compounds from TCM showing Bitter Flavor property. A pharmacophore model can be considered as the highest common denominator of a group of molecules exhibiting a similar pharmacological profile and which are recognized by the same site of the target protein. HipHop algorithm has been used to build pharmacophore of T2R1, T2R10, T2R14, and T2R46, which attempts to produce an alignment of compounds expressing certain activity against a particular target and by superposition of diverse conformations to find common three-dimensional arrangements of features shared between them ${ }^{18}$. The best pharmacophore model of T2Rs have been built to search Traditional Chinese Medicine Database (TCMD) (Version 2009), questing for the agonists from traditional Chinese medicine for further research of the connection between the "Bitter Flavor" and T2Rs.

\section{Generation of common feature pharmacophore model}

\section{A. Compounds and biological data}

The studies were implemented on a series of T2R1 agonists reported by literature ${ }^{15,19-21}$. To generate the pharmacophore model, these compounds were selected as the training set 
(Figure 2) according to the following criteria: a). Sharing certain structural diversity; b). High antagonistic activity in each series; c). Containing similar pharmacophore features to ensure their similar binding models. Considering the distribution of structural diversity, twelve compounds were selected to generate the pharmacophore model and the other compounds were used as test set to validate the model. The agonists of T2R10, T2R14 and T2R46 have been listed in Supplementary Materials.

\section{B. Pharmacophore generation and validation}

In this study, the main steps ${ }^{21,22}$ of pharmacophore generation are as follows $s^{22,23}$ :

\section{Conformational Analysis}

The 3D qualitative pharmacophore hypotheses have been constructed by HipHop (Common Feature Pharmacophore Generation) within Accelrys Discovery Studio 4.0 (DS4.0). Ligand conformations were created within the relative energy threshold of $20 \mathrm{kcal} / \mathrm{mol}$ by the BEST mode (Best Quality Conformer Generation) at the number of 255 maximum conformations.

\section{Common Feature Mapping}

According to the Feature Mapping's initial analysis, hydrogen bond acceptor (A), hydrogen bond donor (D), hydrophobic $(\mathrm{H})$, and ring aromatic $(\mathrm{R})$, which well-mapped all of the training set ligands, has been selected during the pharmacophore generation. These pharmacophore features can characterize the interaction between the ligand and receptor.

\section{Pharmacophore Generation and Verification}

The HipHop algorithm attempts to produce an alignment of compounds by superposition of diverse conformations to find common three-dimensional arrangements of features shared 
between them. The Minimum Features has been set as 3, while the Maximum Features was 10. And the maximum number of pharmacophores generated was set as 10 .

In this study, external test set molecules were used for validating the pharmacophore hypotheses, which have not been used for pharmacophore model generation. A test database of experimentally known T2Rs agonists embedded in a database consisting of some drug-like molecules (taken from the MDL MDDR database: Version2007.2) was constructed to evaluate all of the pharmacophore models. All of the identified ligands were filtered by Lipinskis Rule and were similar in chemical structural characteristics. To evaluate the performance of the models, four parameters (i.e., A\%, Y\%, N, and CAI) and the relationship between them was revealed in Figure 3 with the correlativity as follows ${ }^{22}$ :

$$
\begin{aligned}
& \mathrm{A} \%=\frac{\mathrm{Ha}}{\mathrm{A}} \times 100 \% \\
& \mathrm{Y} \%=\frac{\mathrm{Ha}}{\mathrm{Ht}} \times 100 \% \\
& \mathrm{~N}=\frac{\mathrm{Ha} \times \mathrm{D}}{\mathrm{Ht} \times \mathrm{A}} \\
& \mathrm{CAI}=\mathrm{N} \times \mathrm{A} \%
\end{aligned}
$$


A\% can represent the ability to identify active compounds from the test database and Y\% represents the proportion of active compounds in hit compounds. $\mathrm{N}$, the index of effective identification, is used to evaluate the ability of the models to identify active compounds from non-active compounds. CAI, a comprehensive evaluation index, is used to identify the best pharmacophore model. $\mathrm{D}$ is the total number of compounds in the test database and $\mathrm{A}$ is the number of active compounds. Ht is the total number of hit compounds from the test database and $\mathrm{Ha}$ is the number of active hit compounds from the test database. The model with the highest value of CAI is considered to be the best.

\section{Virtual screening}

According to the performance in terms of the enrichment factor (CAI value) of pharmacophore models, the most excellent pharmacophore model can be useful filters for virtual screening to identify T2R1, T2R10, T2R14 and T2R46 agonists within large compound repositories in TCM. This research select the model with highest CAI value served as a query to perform $3 D$ Flexible Searching operation in DS 4.0 to search Traditional Chinese Medicine Database (TCMD, version 2009), which contains 23033 natural compounds from 6735 medicinal plants. Moreover, all potential hit compounds in the database should be satisfied the Lipinski's rule of five requirements ${ }^{24,25}$.

\section{Results}

\section{Pharmacophore Models Generation}

Twelve compounds were used as the training set for a HipHop running. The top 10 pharmacophore models with the calculation results are detailed in Table 2. According to the simulated results, the main features including Hydrophobic $(H)$ and H-Bond Acceptors $(A)$ 
have been generated. Both of the Direct Hi and Partial Hit values of these 10 pharmacophore models are "111111" and "000000", which confirm that all of the eleven molecules in training set have been taken into the generation of the models. The "4" value of Max Fit proves to us that all of the features in models can match with the molecules in training set. The Rank scores indicate the matching degree between the pharmacophore feature and the molecules. In general, the higher the score, the model matched more satisfactorily. The pharmacophore model calculation results of T2R10, T2R14 and T2R46 have been listed in Supplementary Materials.

\section{Pharmacophore Models Validation}

On the basis of the diagram of indicators evaluating the pharmacophore models showed before, the parameter values for each pharmacophore model were showed in Table 3. NO.1 MODEL with the highest CAI value has been selected to screen TCMD2009 database. The pharmacophore feature of Model 1 mapped with ligand Xanthohumol has been showed in Figure 4. The parameter values for T2R10, T2R14 and T2R46 pharmacophore models have been listed in Supplementary Materials.

\section{Virtual screening}

To quest for the potential agonists of T2R1, T2R10, T2R14 and T2R46, the pharmacophore model generated by HipHop was used as the query to perform a search of all of the known compounds from TCMD2009. According to the results of virtual screening, 2173 compounds from 206 Chinese Herbs have been hit as T2Rs' agonists and $71.84 \%$ of which are belong to the Bitter Flavor property. The partial virtual screening hits were showed in Table 4. Furthermore, the experimental evidence of the hits through virtual screening was validated via literature mining. Andrographolide could activate T2R46, while Amarogentin and Strychnine could both activate T2R10 and T2R $46^{26}$. Moreover, Ginkgolide A is recorded 
as bitter crystals from ethanol in Merck Index ${ }^{15}$ although there is no very definite target being studied. The reported literature could prove the reliability of pharmacophore-based virtual screening to a certain extent, which indicates that further biological activity experiment is worth being studied.

\section{Discussion}

\section{Correlation analysis between Bitter Flavor and T2Rs}

According to the simulation results, $71.84 \%$ agonists of $\mathrm{T} 2 \mathrm{Rs}$ are derived from medicinal herbs showing Bitter Flavor property, such as Salvia miltiorrhiza Bunge. , Coptis chinensis Franch. Rheum palmatum $L$ and so on. It indicated that the pharmacophore models of T2Rs can gather the same structural characteristics and are capable to identify the compounds drawing from TCM showing Bitter Flavor property. From this point can be seen that there is a strong correlation between T2Rs and Bitter Flavor property. The hypothesis would be confirmed that traditional Chinese Bitter Flavor theory having instructive significance on account of the relation between TCM showing Bitter Flavor property and T2Rs. Furthermore, the hits through virtual screening were reported showing multi-targets effects which were identical with those reported in literature ${ }^{27}$. It suggested that their side effects may be caused by non-selective property of T2Rs, which could reveal the correlation between T2Rs and Bitter Flavor theory.

\section{Bitter Flavor describes more than taste feeling}

As we showed before, 'flavor' describes more than the taste feeling of drugs but also the effect of drugs on man. Similar function is grouped and described by one taste property, such as 'bitter' or 'pungent'. Similarly, T2Rs activation is more than bitter taste function as we listed in Introduction. Recent literature suggests that the bitter taste receptor agonist quinine 
reduces calorie intake and increases the post-prandial release of cholecystokinin in healthy subjects $^{28}$. Berberine, the agonist of T2R38 induces GLP-1 secretion through activation of bitter taste receptor pathways ${ }^{29}$ as well as a bitter herbal medicine Gentiana scabra root $^{30}$. And bitter taste receptors can influence glucose homeostasis ${ }^{31}$. Moreover, it is reported that tumor necrosis factor a potent pro-inflammatory cytokine, is preferentially expressed in a subset of taste bud cells to regulate bitter taste response, which may contribute to some chronic inflammatory diseases ${ }^{32}$. Same with Bitter Flavor theory, the medical herbs showing Bitter Flavor property have other functions such as reducing blood glucose, anti-inflammatory effect and so on. To great extent, it positively confirmed the hypothesis that traditional Chinese Bitter Flavor theory having instructive significance on account of the relation with bitter taste receptors.

\section{Method limitation analysis}

In this paper, four subtypes of bitter taste receptors (T2R1, T2R10, T2R14 and T2R46) were studied through virtual screening based on pharmacophore models to explore the relationship with Bitter Flavor. However, there are 25 subtypes are included in T2R family. Therefore, this study reveals the relationship between T2Rs family and Bitter Flavor property to a certain extent while more data should be added in further study.

\section{Conclusions}

In this paper, ligand-based pharmacophore models of T2R1, T2R10, T2R14 and T2R46 have been generated to screen agonists from TCM. According to the results of virtual screening, 2173 compounds from 206 Chinese Herbs have been hit and $71.84 \%$ of which are belong to the Bitter Flavor property. The results show that T2Rs agonist pharmacophore models are capable of accumulating bitter herbs and identify the effective components from 
herbs showing Bitter Flavor property, which provides supporting evidence for the hypothesis that Bitter Flavor having instructive significance on account of the relation with bitter taste receptors. The method used in this paper provides a way for exploring the scientific connotation of Five Flavors theory, which can be extended to solve similar problems in TCM theory.

\section{Abbreviation}

TCM Traditional Chinese Medicine

GPCR G Protein Coupled Receptor

T2Rs Taste Type II Receptors

TCMD Traditional Chinese Medicine Database

DS4.0 Accelrys Discovery Studio 4.0

T2R1 Taste receptor type 2 member 1

T2R10 Taste receptor type 2 member 10

T2R14 Taste receptor type 2 member 14

T2R46 Taste receptor type 2 member 46

\section{Author Contributions}

Y-J Qiao and Y-L Zhang have been conceived and designed the experiments. Y-X Zhang and X Wang have been involved in processing data and preparing the manuscript. S-F Wang participated in the discussion of views in the paper. All authors have read and approved the final manuscript. 


\section{Acknowledgements}

This study was financially supported: National Science Foundation of China: Investigation of chemical components and biological mechanisms of spicy and bitter Chinese herbal medicine for promoting blood circulation and removing blood stasis (Project No. 81430094); Beijing Municipal Natural Science Foundation (Project No. 7164239); Special issue of traditional Chinese Medicine Research in the capital of China (Project No. 16ZY11). Beijing University of Chinese Medicine: Graduate student independent subject (Project No. 1000061220956)

\section{References}

1 Fu, J. L., Pang, J. X., Zhao, X. L. \& Han, J. X. The Quantitative Ideas and Methods in Assessment of Four Properties of Chinese Medicinal Herbs. Cell Biochem Biophys 71, 1307-1312 (2015).

2 Leung, K.-f. et al. Development and validation of the Chinese Quality of Life Instrument. Health and quality of life Outcomes 3, 26 (2005).

$3 \mathrm{Xu}$, L. et al. Developing novel and general descriptors for traditional Chinese medicine (TCM) formulas: A case study of quantitative formula-activity relationship (QFAR) model for hypertension prescriptions. Chemometr Intell Lab 109, 186-191 (2011).

4 Wang, X. et al. Study on relations between transient receptor potential vanilloid 1 and pungent property of traditional Chinese medicines. Zhongguo Zhong yao za zhi= Zhongguo zhongyao zazhi= China journal of Chinese materia medica 39, 2422-2427 (2014).

5 Zhang, X. \& Wang, Z. A discussion on the reason of differences between property and flavor of Chinese Materia Medica of ancient and modern times. Zhonghua yi shi za zhi (Beijing, China: 1980) 39, 164-167 (2009).

6 Ni, X., Han, X., Yin, D. \& Liu, C. Study on medication regularity of traditional Chinese medicines in treating attention deficit hyperactivity disorder based on data mining. Zhongguo Zhong yao za zhi= Zhongguo zhongyao zazhi= China journal of Chinese materia medica 40, 1185-1191 (2015).

7 Finger, T. E. \& Kinnamon, S. C. Taste isn't just for taste buds anymore. F1000 biology reports 3 (2011).

8 Deshpande, D. A. et al. Bitter taste receptors on airway smooth muscle bronchodilate by localized calcium signaling and reverse obstruction. Nature Medicine 16, 
1299-U1298 (2010).

9 Grassin-Delyle, S., Naline, E. \& Devillier, P. Taste receptors in asthma. Current opinion in allergy and clinical immunology 15, 63-69 (2015).

10 Robinett, K. S. et al. Bitter taste receptor function in asthmatic and nonasthmatic human airway smooth muscle cells. American journal of respiratory cell and molecular biology 50, 678-683, doi:10.1165/rcmb.2013-0439RC (2014).

11 Depoortere, I. Taste receptors of the gut: emerging roles in health and disease. Gut 63, 179-190, doi:10.1136/gutjnl-2013-305112 (2014).

12 Sanderson, M. J. \& Madison, J. M. Bitter treats for better breathing. Nature Medicine 16, 1190-1191 (2010).

13 Manson, M. L. et al. Bitter taste receptor agonists mediate relaxation of human and rodent vascular smooth muscle. European journal of pharmacology 740, 302-311, doi:10.1016/j.ejphar.2014.07.005 (2014).

14 Levit, A. et al. The bitter pill: clinical drugs that activate the human bitter taste receptor TAS2R14. Faseb J 28, 1181-1197 (2014).

15 Wiener, A., Shudler, M., Levit, A. \& Niv, M. Y. BitterDB: a database of bitter compounds. Nucleic acids research 40, D413-D419 (2012).

16 Mannhold, R., Kubinyi, H., Folkers, G., Langer, T. \& Hoffmann, R. D. Pharmacophores and pharmacophore searches. Vol. 32 (John Wiley \& Sons, 2006).

17 Guner, O. F. Pharmacophore perception, development, and use in drug design. International University Line, La Jolla, CA 29 (2000).

18 Poptodorov, K., Luu, T. \& Hoffmann, R. D. Pharmacophore model generation software tools. Methods and Principles in Medicinal Chemistry 32, 17 (2006).

19 Intelmann, D. et al. Three TAS2R bitter taste receptors mediate the psychophysical responses to bitter compounds of hops (Humulus lupulus L.) and beer. Chemosensory Perception 2, 118-132 (2009).

20 Kohl, S., Behrens, M., Dunkel, A., Hofmann, T. \& Meyerhof, W. Amino Acids and Peptides Activate at Least Five Members of the Human Bitter Taste Receptor Family. J Agr Food Chem 61, 53-60 (2013).

21 Brockhoff, A. et al. Receptor Agonism and Antagonism of Dietary Bitter Compounds. Journal of Neuroscience 31, 14775-14782 (2011).

22 Wang, X., Xiang, Y. H., Ren, Z. Z., Zhang, Y. L. \& Qiao, Y. J. Rational questing for inhibitors of endothelin converting enzyme-1 from Salvia miltiorrhiza by combining ligand- and structure-based virtual screening. Can J Chem 91, 448-456 (2013).

23 He, Y., Jiang, L., Yang, Z., Qiao, Y. \& Zhang, Y. A combination of pharmacophore modeling, molecular docking, and virtual screening for P2Y12 receptor antagonists 
from Chinese herbs. Canadian Journal of Chemistry (2014).

24 Lipinski, C. A. Drug-like properties and the causes of poor solubility and poor permeability. Journal of pharmacological and toxicological methods 44, 235-249 (2000).

25 Bhal, S. K., Kassam, K., Peirson, I. G. \& Pearl, G. M. The Rule of Five revisited: applying $\log \mathrm{D}$ in place of $\log \mathrm{P}$ in drug-likeness filters. Molecular pharmaceutics $\mathbf{4}$, 556-560, doi:10.1021/mp0700209 (2007).

26 Meyerhof, W. et al. The molecular receptive ranges of human TAS2R bitter taste receptors. Chemical senses 35, 157-170, doi:10.1093/chemse/bjp092 (2010).

27 Clark, A. A., Liggett, S. B. \& Munger, S. D. Extraoral bitter taste receptors as mediators of off-target drug effects. Faseb J 26, 4827-4831 (2012).

28 Andreozzi, P. et al. The bitter taste receptor agonist quinine reduces calorie intake and increases the postprandial release of cholecystokinin in healthy subjects. Journal of neurogastroenterology and motility 21, 511-519 (2015).

29 Yu, Y. et al. Berberine induces GLP-1 secretion through activation of bitter taste receptor pathways. Biochemical pharmacology (2015).

30 Suh, H.-W. et al. A bitter herbal medicine Gentiana scabra root extract stimulates glucagon-like peptide-1 secretion and regulates blood glucose in $\mathrm{db} / \mathrm{db}$ mouse. Journal of ethnopharmacology 172, 219-226 (2015).

31 Dotson, C. D. et al. Bitter taste receptors influence glucose homeostasis. PloS one 3 (2008).

32 Feng, P. et al. Regulation of bitter taste responses by tumor necrosis factor. Brain, behavior, and immunity (2015). 
Figure 1 Taste Type II Receptors and Specific Ligands Distribution

Figure 1 Chemical structure of T2R1 agonists

Figure 2 Schematic diagram of indicators evaluating the pharmacophore models

Figure 3 T2R1 Pharmacophore Model_01 features (A) and the matching map with ligand Xanthohumol (B) 



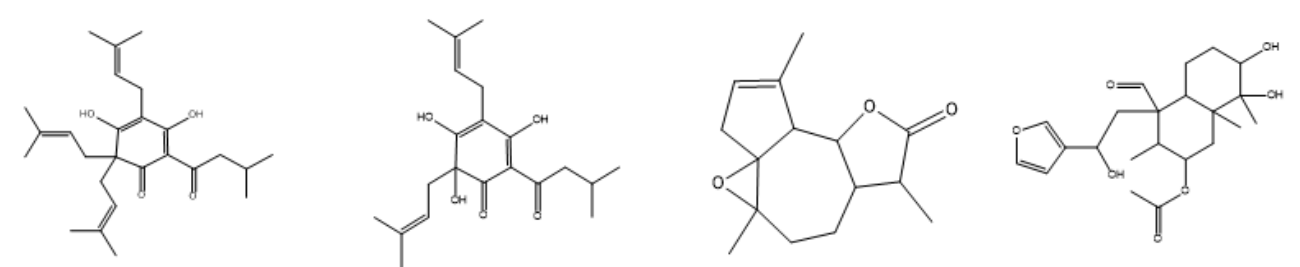

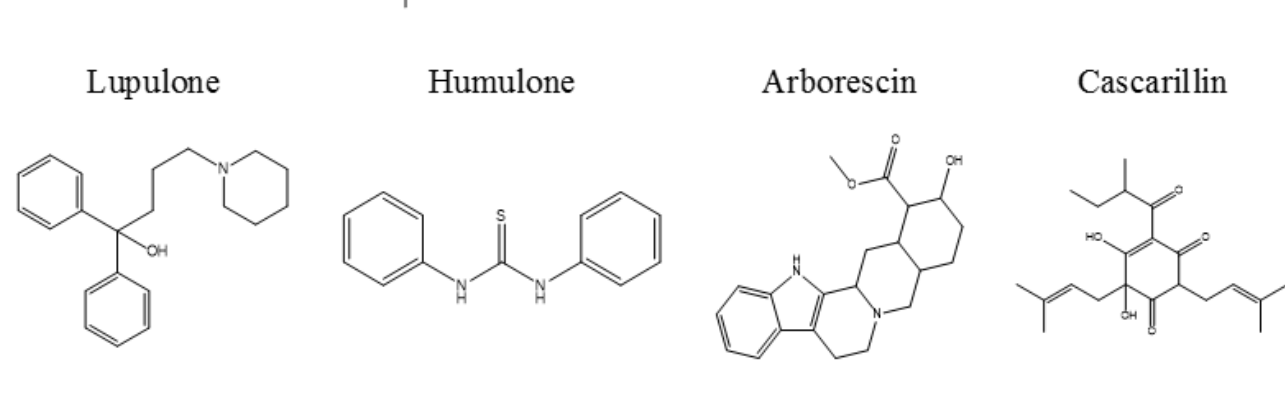

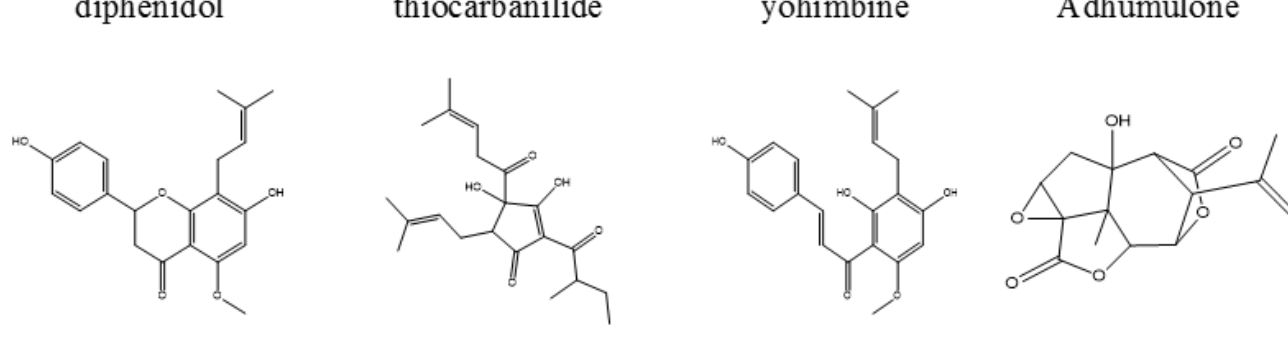




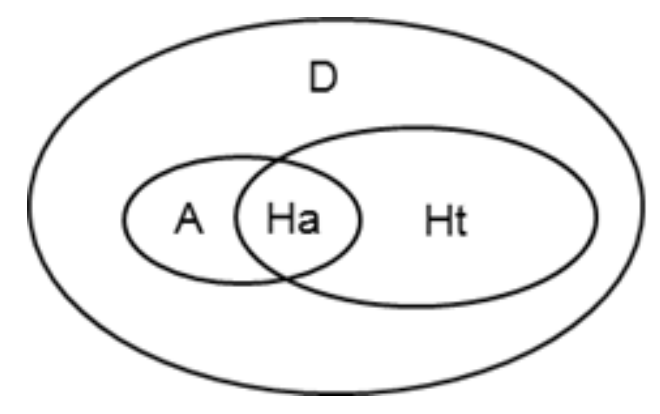



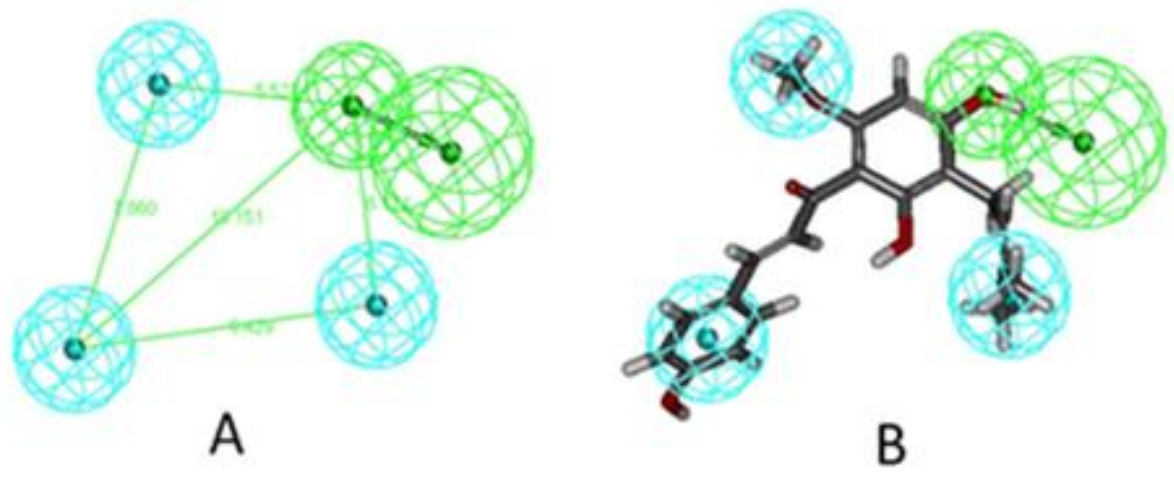


\section{Tables}

Table 1 Natural agonists of T2Rs and TCM sources

\begin{tabular}{|c|c|c|c|}
\hline Compounds & TCM Source & Flavor Property & T2Rs \\
\hline Benzoin & Benzoinum & Bitter/Pungent & T2R10, T2R14 \\
\hline Andrographolide & Herba Andrographis & Bitter & T2R46, T2R47, T2R50 \\
\hline Sinigrin & Semen Lepidii & Pungent/Bitter & T2R16, T2R38 \\
\hline Xanthohumol & Flos Humuli Lupuli & Bitter/Pungent & $\mathrm{T} 2 \mathrm{R} 1, \mathrm{~T} 2 \mathrm{R} 14, \mathrm{~T} 2 \mathrm{R} 40$ \\
\hline Adhumulone & Flos Humuli Lupuli & Bitter/Pungent & $\mathrm{T} 2 \mathrm{R} 1, \mathrm{~T} 2 \mathrm{R} 40$ \\
\hline Adlupulone & Flos Humuli Lupuli & Bitte/Pungent & T2R1, T2R14 \\
\hline Absinthin & Radix Stephaniae Tetrandrae & Bitter/Pungent & T2R10, T2R14, T2R46, T2R47 \\
\hline Quassin & Cortex Meliae & Bitter & T2R4, T2R10, T2R14, T2R46, T2R47 \\
\hline Amarogentin & Semen Armeniacae Amarum & Bitter & T2R1, T2R4, T2R16, T2R39, T2R43, T2R46, T2R47, T2R50 \\
\hline Quinine & Cortex Cinchonae & Bitter & T2R4, T2R7, T2R10, T2R14, T2R39, T2R40, T2R43, T2R44, T2R46 \\
\hline Aloin & Herba Aloes & Bitter & $\mathrm{T} 2 \mathrm{R} 43, \mathrm{~T} 2 \mathrm{R} 44$ \\
\hline Aristolochic acid & Fructus Aristolochiae & Bitter & $\mathrm{T} 2 \mathrm{R} 14, \mathrm{~T} 2 \mathrm{R} 43, \mathrm{~T} 2 \mathrm{R} 44$ \\
\hline Brucine & Semen Strychni & Bitter & $\mathrm{T} 2 \mathrm{R} 4, \mathrm{~T} 2 \mathrm{R} 46$ \\
\hline Picrotoxinin & Radix Stephaniae Tetrandrae & Bitter/Pungent & T2R10, T2R14, T2R46, T2R47 \\
\hline Strychnine & Semen Strychni & Bitter & $\mathrm{T} 2 \mathrm{R} 10, \mathrm{~T} 2 \mathrm{R} 46$ \\
\hline
\end{tabular}


Table 2 T2R1 Pharmacophore model calculation results

\begin{tabular}{llllll}
\hline Model & Features & Rank & Direct Hit & Partial Hit & Max Fit \\
\hline 01 & HHHA & 50.372 & 111111 & 000000 & 4 \\
02 & HHHA & 50.260 & 111111 & 000000 & 4 \\
03 & HHHA & 49.553 & 111111 & 000000 & 4 \\
04 & HHHA & 49.285 & 111111 & 000000 & 4 \\
05 & HHHA & 49.096 & 111111 & 000000 & 4 \\
06 & HHHA & 48.796 & 111111 & 000000 & 4 \\
07 & HHHA & 48.796 & 111111 & 000000 & 4 \\
08 & HHHA & 48.261 & 111111 & 000000 & 4 \\
09 & HHHA & 48.105 & 111111 & 000000 & 4 \\
10 & HHHA & 47.887 & 111111 & 000000 & 4 \\
\hline
\end{tabular}

Table 3 T2R1 Parameter values for each pharmacophore model

\begin{tabular}{cccccccc}
\hline Model & $\mathbf{A}^{\mathbf{a}}$ & $\mathbf{D}^{\mathbf{b}}$ & $\mathbf{H t}^{\mathbf{c}}$ & $\mathbf{H a}^{\mathbf{d}}$ & $\mathbf{A \%}^{\mathbf{e}}$ & $\mathbf{N}^{\mathbf{f}}$ & $\mathbf{C A I}^{\mathbf{g}}$ \\
\hline $\mathbf{1}$ & $\mathbf{2 7}$ & $\mathbf{1 8 0}$ & $\mathbf{4 1}$ & $\mathbf{1 9}$ & $\mathbf{7 0 . 4}$ & $\mathbf{3 . 0 9}$ & $\mathbf{2 . 1 7}$ \\
2 & 27 & 180 & 46 & 17 & 63.0 & 2.46 & 1.55 \\
3 & 27 & 180 & 46 & 18 & 66.7 & 2.61 & 1.74 \\
4 & 27 & 180 & 48 & 17 & 63.0 & 2.36 & 1.49 \\
5 & 27 & 180 & 42 & 16 & 59.3 & 2.54 & 1.50 \\
6 & 27 & 180 & 45 & 18 & 66.7 & 2.67 & 1.78 \\
7 & 27 & 180 & 47 & 18 & 66.7 & 2.55 & 1.70 \\
8 & 27 & 180 & 46 & 18 & 66.7 & 2.61 & 1.74 \\
9 & 27 & 180 & 49 & 17 & 63.0 & 2.31 & 1.46 \\
10 & 27 & 180 & 59 & 16 & 59.3 & 1.81 & 1.07 \\
\hline
\end{tabular}

${ }^{\mathrm{a}} \mathrm{A}$ is the number of active compounds; ${ }^{\mathrm{b}} \mathrm{D}$ is the number of compounds in the test database; ${ }^{\mathrm{c}} \mathrm{Ht}$ is the number of hits using pharmacophores to search; ${ }^{\mathrm{d}} \mathrm{Ha}$ is the number of active hits using pharmacophores to search; ${ }^{\mathrm{e}} \mathrm{A} \%$ represents the ability to identify active compounds from the test database; ${ }^{\mathrm{f}} \mathrm{N}$ represents the ability to identify active compounds from nonactive compounds; ${ }^{\mathrm{g}} \mathrm{CAI}$ is the comprehensive appraisal index. 
Table 4 Partial Virtual Screening Hits of T2Rs Agonists

\begin{tabular}{|c|c|c|c|c|}
\hline Name & Structure & TCM Souces & Flavors & T2Rs \\
\hline Ginkgolide A & & Semen Ginkgo & Sweet/Bitter & $\mathrm{T} 2 \mathrm{R} 10, \mathrm{~T} 2 \mathrm{R} 14$ \\
\hline Ginkgolide B & & Semen Ginkgo & Sweet/Bitter/ & T2R10, T2R14 \\
\hline Albiflorin & & Radix Paeoniae Alba & Bitter/Sour & $\mathrm{T} 2 \mathrm{R} 10, \mathrm{~T} 2 \mathrm{R} 14$ \\
\hline Albiflorinr1 & & Radix Paeoniae Alba & Bitter/Sour & $\mathrm{T} 2 \mathrm{R} 10, \mathrm{~T} 2 \mathrm{R} 14$ \\
\hline Saikosaponin F & & Radix Bupleuri & Bitter & $\mathrm{T} 2 \mathrm{R} 10, \mathrm{~T} 2 \mathrm{R} 14, \mathrm{~T} 2 \mathrm{R} 46$ \\
\hline
\end{tabular}




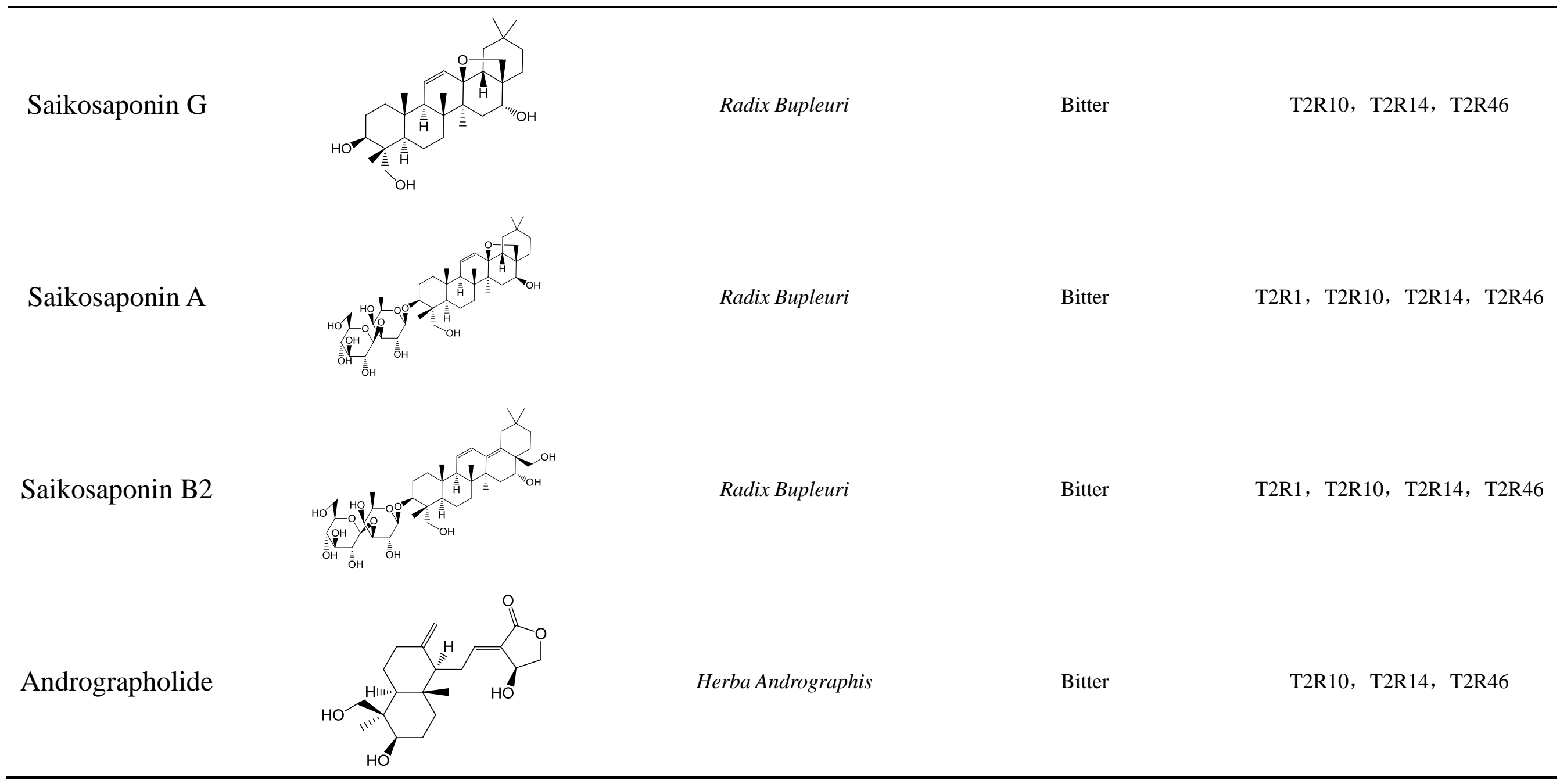




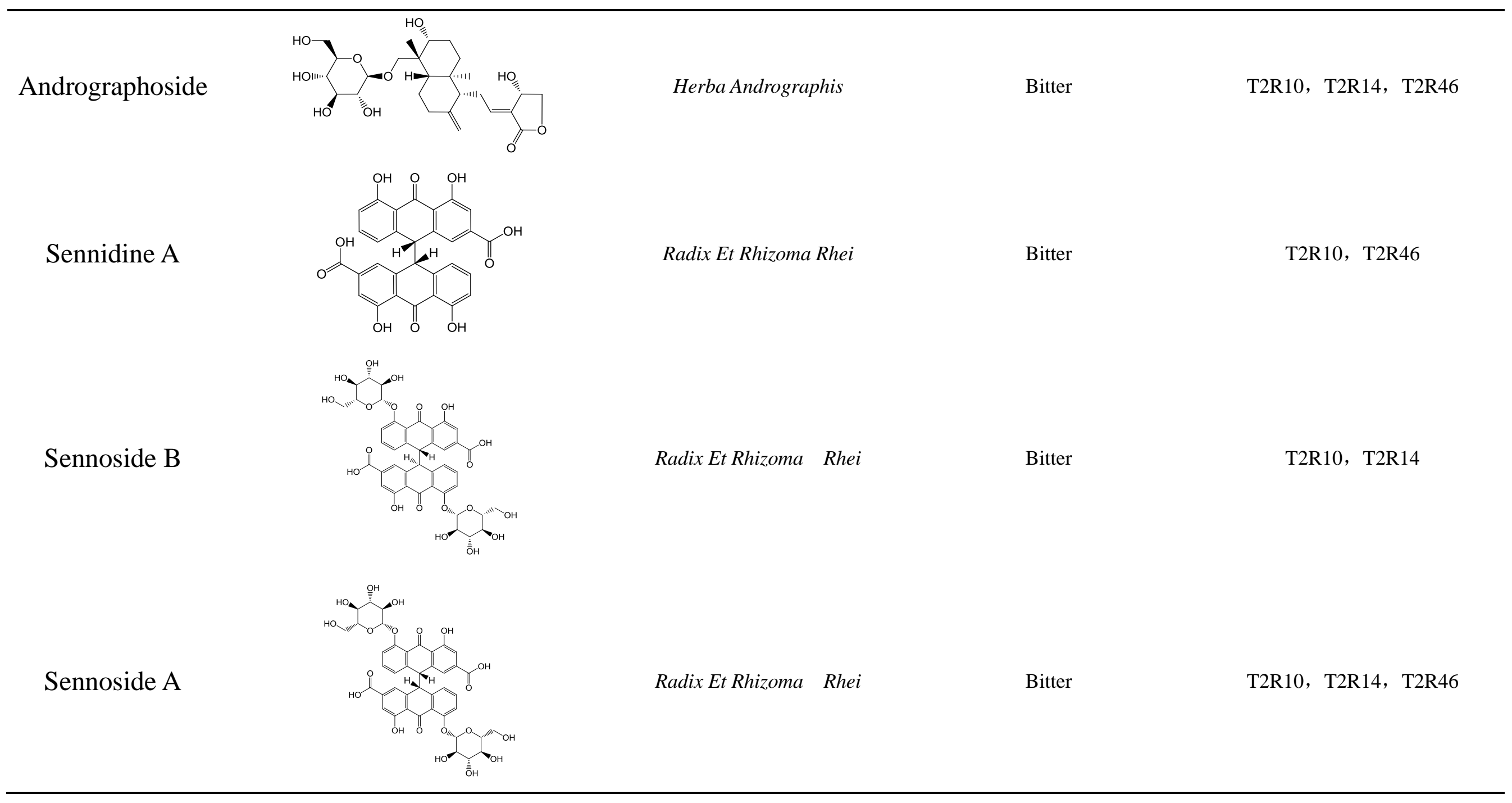




T2R1, T2R10, T2R46
Sennidine A
Salvianolic Acid
R




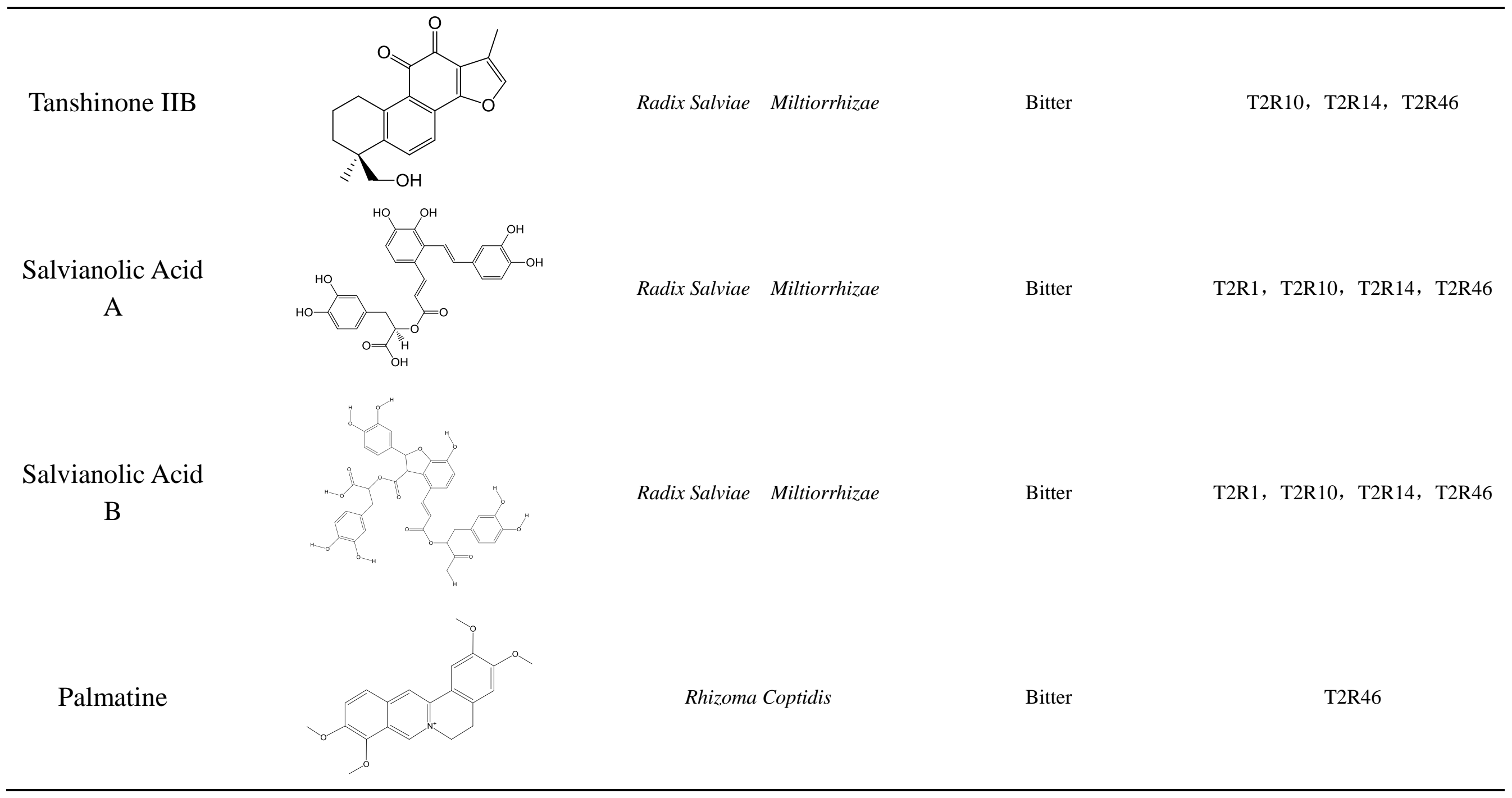




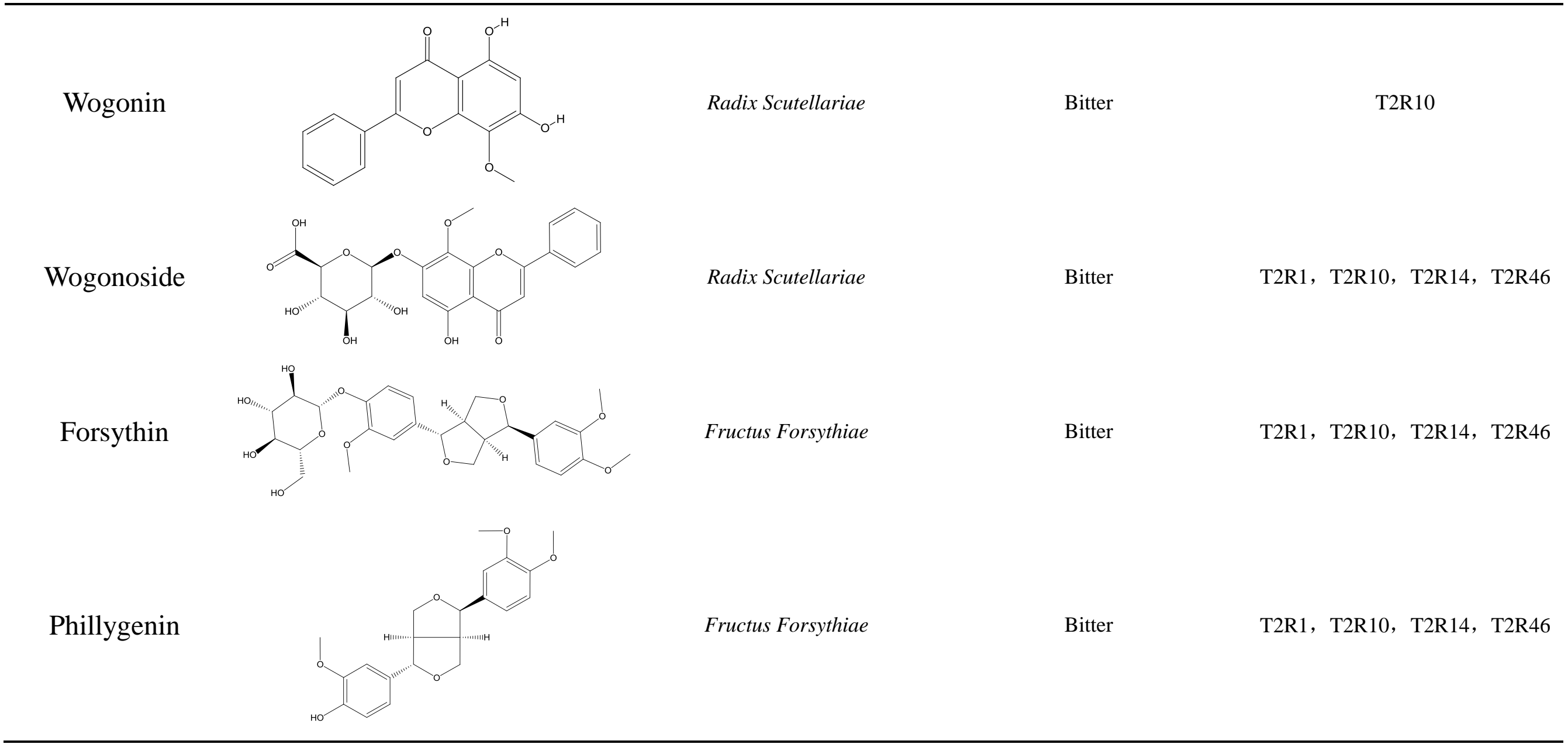




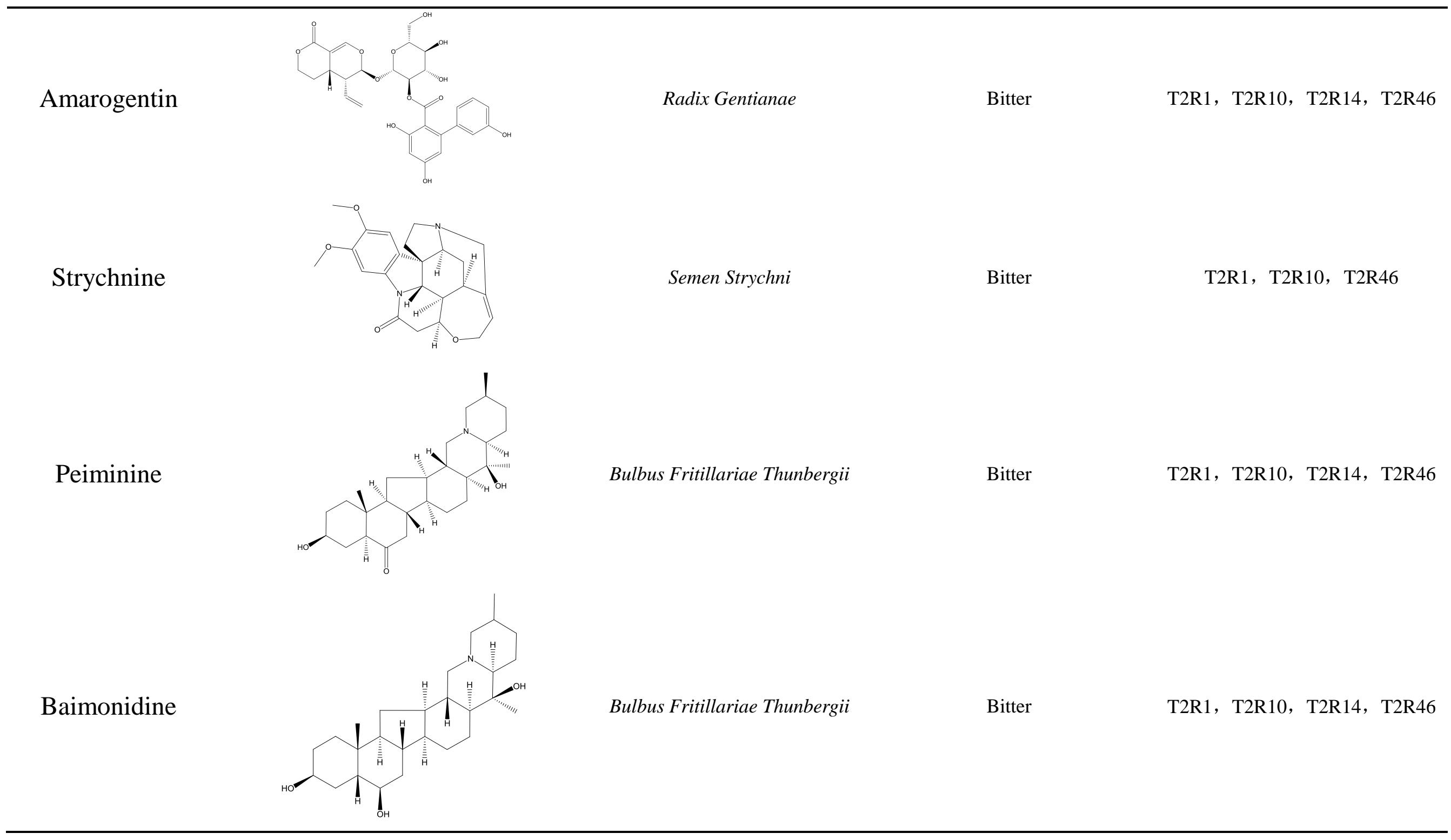




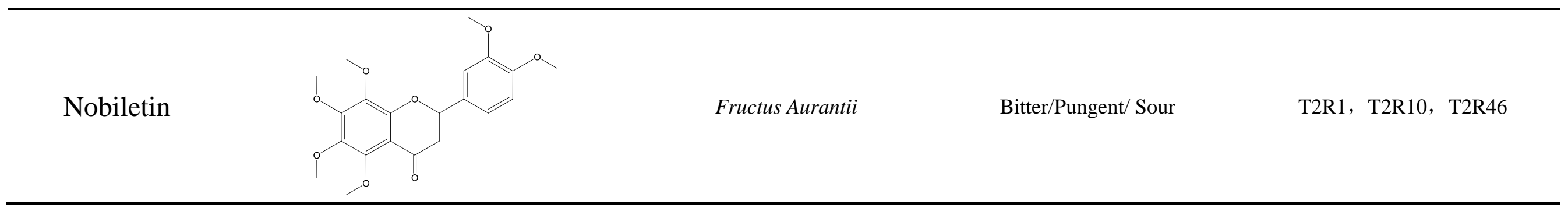

